\title{
Development of four EST-SSR multiplex PCRs in the Pacific oyster (Crassostrea gigas) and their validation in parentage assignment
}

\author{
Ronghua $\mathrm{Li}^{\mathrm{a}, \mathrm{b}}$, Qi Li ${ }^{\mathrm{a}}$, Florence Cornette ${ }^{\mathrm{b}}$, Lionel Dégremont ${ }^{\mathrm{b}}$ and Sylvie Lapègue ${ }^{\mathrm{b}, ~ *}$ \\ ${ }^{a}$ Fisheries College, Ocean University of China, Qingdao 266003, China \\ ${ }^{\mathrm{b}}$ Ifremer, Laboratoire de Génétique et Pathologie, 17390 La Tremblade, France \\ *: Corresponding author : Sylvie Lapègue, Tel.: +33 5467626 31; fax: +33 5467626 11, email address : \\ slapegue@ifremer.fr
}

\begin{abstract}
:
We report four highly informative multiplex PCRs developed from 12 previously described EST-SSRs in Crassostrea gigas. We evaluated and validated these multiplex PCRs in 12 full-sib families. The average allelic richness and the polymorphism information content (PIC) were 11.1 and 0.811 respectively. The combined power of exclusion was greater than $99.99 \%$ using all four multiplex assays. A hundred and forty three tests of segregation ratios revealed 11 significant departures from expected Mendelian ratios. The frequency of null alleles was estimated as $4.9 \%$ of all the alleles segregating based on a within-family analysis of Mendelian segregation patterns. Parentage analysis of real offspring demonstrated that $97 \%$ of all offspring were unambiguously allocated to a pair of parents based on two multiplex PCRs with only a $4 \%$ error rate, and $100 \%$ of the offspring were correctly allocated to their parents when three multiplex PCRs were used.
\end{abstract}

Keywords: Pacific oyster; Crassostrea gigas; Microsatellite; EST; Multiplex PCR; Parentage assignment 


\section{Introduction}

The Pacific oyster, Crassostrea gigas, is the main cultivated oyster species worldwide with a global production of 4.2 million metric tons in 2007 (FAO, 2010). A great deal of genetic research has been conducted to improve the production of this species (Camara et al., 2008; Dégremont et al., 2007; Desrosier et al., 1993; Evans and Langdon, 2006; Guo et al., 1994; Hubert and Hedgecock, 2004; Langdon et al., 2003; Li et al., 2006; Sauvage et al., 2010; Tanguy et al., 2008). In particular, significant mortality of the $C$. gigas has been reported during the summer months in Japan, USA and France. (Mori, 1979; Perdue and Erickson, 1984; Samain and McCombie, 2008), and survival of juvenile Pacific oysters under summer mortality conditions has a strong genetic basis (Dégremont et al., 2007), which was successfully confirmed over several generations through a divergent selection (Dégremont et al., 2010). The genetic improvement based on parentage selection and marker assisted selection was considered to be one of the methods to reduce the mortality of this species and consequently increase the production.

Simple sequence repeats (SSRs; also called microsatellites) have been growing in popularity in genetic analysis because of their high level of polymorphism and codominant characterization. The effectiveness of the SSRs in parentage analysis of $C$. gigas has been proved (Boudry et al., 2002; Li R et al., 2009; Matson et al., 2008). Simple sequence repeats derived from expressed sequence tag (EST) databases (EST-SSRs) have a number of advantages, such as higher transferability across a broader taxonomic range, lower frequencies of null alleles, and association with known function genes (Liu et al., 1999; Pashley et al., 2006). Thus they could be valuable for studies of local adaptation, population structure, selective breeding programs, parentage analysis and genome mapping.

The multiplex PCR technique not only reduces the time and cost associated with SSRs analysis, but also decreases the repeated manipulation of large numbers of sample during the genotyping and, therefore, the risk of handling errors (Porta et al., 2006). However, in C. gigas, only one multiplex developed from genomic DNA was reported (Taris et al., 2005).

In this paper, we first reported the development of multiplex PCRs from previously described EST-SSRs of $C$. gigas and their power in parentage assignment was validated in twelve single-pair mating families.

\section{Material and methods}

Twelve C. gigas single-pair mating families were produced from genitors selected for summer mortality or reproduction behavior in February 2009 in Ifremer La Tremblade, France. They were used to test the resolving power of the four EST-SSR multiplex PCRs. For each family, gill samples of both parents and 23 offspring, randomly sampled at one year old, were stored in 70\% ethanol until DNA extraction. Genomic DNA was extracted from gill tissue by the chloroform/isoamylalcohol method and purified with the DNA Clean Up System (Promega, Madison, WI, USA). Quality and concentration of each DNA sample were assessed using a spectrophotometer and by running a small amount on a $1 \%$ agarose gel.

Eleven sets of primers were selected from previously described expressed sequence tagderived SSRs (Li Q et al., 2009; Sauvage et al., 2009; Yu and Li, 2007) according to their polymorphism, linkage information, sequence motif repeats and amplification behavior. A SSR developed from C. gigas amylase gene was also incorporated in this study (Sellos et al., 2003). One primer of each pair was labeled with one fluorescent dye (HEX, FAM or NED).

The proper annealing temperature range for each SSR was determined using a panel of 10 individuals. The single PCR mixtures were composed of $1 \cup$ of GoTaq Flexi DNA Polymerase (Promega, Madison, WI, USA), 1× GoTaq Flexi Buffer (Promega, Madison, WI, USA), $2 \mathrm{mM} \mathrm{MgCl}, 0.2 \mathrm{mM}$ of dNTP, $0.24 \mu \mathrm{M}$ of each primer and 16 ng genomic DNA in a final reaction volume of $10 \mu \mathrm{l}$. PCR was performed as follows: an initial denaturation at $95^{\circ} \mathrm{C}$ 
for 2 min was followed by 30 cycles of $95^{\circ} \mathrm{C}$ for $30 \mathrm{~s}, T_{\mathrm{a}}$ (provided by primer synthesis information) for $30 \mathrm{~s}, 72^{\circ} \mathrm{C}$ for $45 \mathrm{~s}$; and final elongation at $72^{\circ} \mathrm{C}$ for $30 \mathrm{~s}$. Products were mixed with formamide and GeneScan 500-ROX size standard (Applied Biosystems, Warrington, UK) respectively, according to the manufacturer's recommendations. After 5 min denaturation followed by rapid cooling, PCR products were detected using an ABI 3130xI Genetic Analyser (Applied Biosystems, Carlsbad, WA, USA), and the fragment length was estimated through the GeneMapper 3.7 software. These loci were then organized into multiplex sets that maximize the number of loci suitable for simultaneous analysis with no allele overlap between loci. Even with the same dye, overlapping is avoided in order to optimize the genotyping.

Primer concentration, annealing temperature and DNA template concentration were then optimized using 10 individuals. PCR multiplex amplifications were conducted using the Typeit Microsatellite PCR Kit (QIAGEN, Hilden, Germany) in $10 \mu$ reaction volumes containing 5 $\mu \mathrm{l}$ of the Type-it Multiplex PCR Master Mix (2x) (including HotStarTaq ${ }^{\circledR}$ Plus DNA Polymerase, Type-it Microsatellite PCR Buffer with $6 \mathrm{mM} \mathrm{MgCl}_{2}$, and dNTPs), $1 \mu \mathrm{l}$ of primer mix (0.05-0.4 $\mu \mathrm{M}$ ), $1 \mu \mathrm{l}$ of genomic DNA (3-10ng), $1 \mu \mathrm{l}$ Q-Solution (5x) and $2 \mu \mathrm{l}$ of RNasefree water. Amplification started with an initial activation step at $95^{\circ} \mathrm{C}$ for $15 \mathrm{~min}$, followed by 30 cycles with denaturation at $94^{\circ} \mathrm{C}$ for $30 \mathrm{~s}, T_{\mathrm{a}}\left(50-62^{\circ} \mathrm{C}\right)$ for $90 \mathrm{~s}$, extension at $72^{\circ} \mathrm{C}$ for $90 \mathrm{~s}$ and final extension at $60^{\circ} \mathrm{C}$ for $10 \mathrm{~min}$. The PCR products were loaded in the ABI 3130xI Genetic Analyser and analyzed as described in single PCR protocol.

The number of alleles $\left(N_{\mathrm{a}}\right)$, polymorphic information content (PIC) and the average nonexclusion probability of each locus in different situation were calculated using Cervus 3.0 (Kalinowski et al., 2007). Allelic Richness $\left(A_{r}\right)$ was estimated with FSTAT 2.9.3 (Goudet, 2001). The genotype data of the progenies in all the 12 single-pair families were pooled together to test the resolve power of the Multiplex PCRs in parentage analysis. The simulation and real parentage assignment were conducted using the likelihood-based approach in CERVUS 3.0. as follows: 10,000 replication cycles, a pool of 24 candidate parents, $100 \%$ of the candidate parents sampled and genotyped, a default typing error rate of $1 \%$ was used. All correctly allocated offspring were included in the Mendelian inheritance analysis at four EST-SSR multiplex PCRs in this study. The chi-square analysis (with $n-1$ degrees of freedom, where $n=$ number of phenotypic classes) was used to measure the goodness-of-fit for expected Mendelian segregation ratios (1:1, 1:2:1, and 1:1:1:1) at the 0.01 probability level.

\section{Results and discussion}

For each single PCR, the range of the suitable annealing temperature was about $\pm 5^{\circ} \mathrm{C}$ around the $T_{\mathrm{a}}$ which was provided by primer synthesis information. Therefore we combined the loci according to a common annealing temperature into four optimized sets of multiplex PCRs presented in Table 1, each of them containing three markers. The optimum DNA template quantity was 3ng which resulted in clearly resolved peaks and unambiguous allele calling in all multiplex reactions. Increasing the template quantity produced interaction effects among fluorochromes, and compromised our ability to reliably score genotypes. The genetic diversity and non-exclusion probabilities for each SSR were also shown in Tables 1 . The combined power of exclusion was greater than $99.99 \%$ across the four panels. The allelic richness per locus ranged from 4 to 16.4, with an average of 11.1. The average PIC was 0.811 from which a high exclusion power was revealed for these Multiplex PCRs in parentage analysis.

The results of Cervus simulations showed that with only two most informative Multiplex PCRs, the total assignment success could be $100 \%$ (Fig 1). In practice, the real parentage analysis performed with 12 C. gigas single-pair mating families demonstrated that $97 \%$ of all offspring were unambiguously assigned to a pair of parents based on the two most informative 
multiplex PCRs with only $4 \%$ error rate comparing with the real family data, and $100 \%$ of the offspring were correctly allocated to their parents when three and four multiplex PCRs were used (Fig 1). The precision of assignment to one correct parental pair depends not only on the number of SSR loci genotyped and their levels of polymorphism but also on the number of potential pairings from which to choose (Matson et al., 2008; Norris et al., 2000). According to simulation, the use of the three multiplex PCRs could also achieve $100 \%$ assignment success when the parents increase to 50 pairs, which was recommended by Bentsen and Olesen (2002) to prevent inbreeding and obtain a long-term response in a mass selection program. Actually, in this study, the assignment success rate still sustains at $97 \%$ when the parents rise up to 800 pairs with the most polymorphic three multiplex PCRs (Fig 2). Thus, two to three multiplex PCRs developed here should be suitable to perform a successful parentage analysis with lower error rate.

The parental genotypes, observed and expected genotypic frequencies of offspring in each family at each of the four multiplex PCRs were shown in Table 2. SSRs developed from C. gigas usually have particularly high frequency null alleles (Li et al., 2003). The presence of null alleles is a classical source of error in parentage assignment with SSRs (Marshall et al., 1998) and it could also bias the estimation of population differentiation (Chapuis and Estoup, 2007). In non-coding genomic SSRs in C. gigas null alleles frequencies have been reported from $11 \%$ to $22 \%$ (Li L et al., 2009; McGoldrick et al., 2000). The frequency of null alleles in EST-SSRs is usually lower than in non-coding genomic SSRs because of lower mutation at splicing sites (Goldstein and Schlotterer, 1999). Yu and Li 2008 reported a null allele frequency of $2.1 \%$ of alleles in 20 EST-derived SSRs of $C$. gigas. Among the 12 loci studied here, null alleles were detected for 3 loci (Cgsili39, CGG008, Cgsili6) based on a withinfamily analysis of Mendelian segregation patterns, 28 of the 576 parental alleles were null alleles, which was $4.9 \%$ of the total alleles (12 loci * 24parents *2). The loci showing null alleles were amplified by single PCRs, which confirmed that they were not caused by the mixture of different primers. Of the 144 genotypic ratios examined (12 families $\times 12$ loci), 1 came from crosses between homozygous parents and thus resulted in offspring identical to the parents or with the expected heterozygote genotype (CGE007 in family LOT 1). Eleven genotypic ratios were still not in agreement with Mendelian segregation after accounting for the presence of null alleles (Table 2) which might reflect the impact of selective breeding process.

In conclusion, the results obtained in this study make the four EST-SSR Multiplex PCRS unique tools in studies of parentage assignment, marker assisted breeding, population genetic analysis and linkage maps of $C$. gigas.

\section{Acknowledgements}

This research has been partially financed by the European Seventh Framework Programme (FP7) through the European Knowledge Based Bio-Economy (KBBE) project REPROSEED (KBBE-3-245119). We also acknowledge the support provided by China Scholarship Council (CSC) to R. Li for her fellowship in Ifremer.

\section{References}

Bentsen, H.B., Olesen, I., 2002. Designing aquaculture mass selection programs to avoid high inbreeding rates. Aquaculture 204, 349-359.

Boudry, P., Collet, B., Cornette, F., Hervouet, V., Bonhomme, F., 2002. High variance in reproductive success of the Pacifc oyster (Crassostrea gigas, Thunberg) revealed by microsatellite-based parentage analysis of multifactorial crosses. Aquaculture 204, 283-296.

Camara, M.D., Evans, S., Langdon, C.J., 2008. Parental relatedness and survival of Pacific Oysters from a naturalized population. J. Shellfish Res. 27(2), 323-336. 
Chapuis, M.P., Estoup, A., 2007. Microsatellite null alleles and estimation of population differentiation. Mol. Biol. Evol. 24(3), 621-631.

Dégremont, L., Bédier, E., Boudry, P., 2010. Summer mortality of hatchery-produced Pacific oyster spat (Crassostrea gigas). II. Response to selection for survival and its influence on growth and yield. Aquaculture 299, 21-29.

Dégremont, L., Ernande, B., Bédier, E., Boudry, P., 2007. Summer mortality of hatcheryproduced Pacific oyster spat (Crassostrea gigas): I. Estimation of genetic parameters for survival and growth. Aquaculture 262, 41-53.

Desrosiers, R.R., Gerard, A., Peignon, J.M., Naciri, Y., Dufresne, L., Morasse, J., Ledu, C., Phelipot, P., Guerrier, P., Dube, F., 1993. A novel method to produce triploids in bivalve molluscs by the use of 6-dimethylaminopurine. J. Exp. Mar. Biol. Ecol. 170, 29-43.

Evans, S., Langdon, C., 2006. Effects of genotype $\times$ environment interactions on the selection of broadly adapted Pacific oysters (Crassostrea gigas). Aquaculture 261, 522-534.

FAO, 2010. World aquaculture production of fish, crustaceans, molluscs, etc., by principal species (ftp://ftp.fao.org/fi/STAT/summary/default. htm\#aqua).

Goldstein, D., Schlotterer, C., 1999. Microsatellites: Evolution and Applications. Oxford University Press, Oxford, UK.

Goudet, J., FSTAT, a Program to Estimate and Test Gene Diversities and Fixation Indices Version 2.9.3. 2001. http://www2.unil.ch/popgen/softwares/fstat.htm Updated from Goudet (1995).

Guo, X., Standish, K., Allen, J.R., 1994. Reproductive potential and genetics of triploid Pacific oysters, Crassostrea gigas (Thunberg). Biol. Bull. 187, 309-318.

Hubert, S., Hedgecock, D., 2004. Linkage Maps of Microsatellite DNA Markers for the Pacific Oyster Crassostrea gigas. Genetics 168, 351-362.

Kalinowski, S.T., Taper, M.L., Marshall, T.C., 2007. Revising how the computer program CERVUS accommodates genotyping error increases success in paternity assignment. Mol. Ecol. 16, 1099-1106.

Langdon, C., Evans, F., Jacobson, D., Blouin, M., 2003. Yields of cultured Pacific oysters Crassostrea gigas Thunberg improved after one generation of selection. Aquaculture 220, 227-244.

Li, G., Hubert, S., Bucklin, K., Ribes, V., Hedgecock, D., 2003. Characterization of 79 microsatellite DNA markers in the Pacific oyster Crassostrea gigas. Mol. Ecol. Notes 3, 228232.

Li, L., Guo, X.M., Zhang, G.F., 2009. Inheritance of 15 microsatellites in the Pacific oyster Crassostrea gigas: segregation and null allele identification for linkage analysis. Chin. J. Oceanol. Limnol. 27, 74-79.

Li, Q., Liu, S.K., Kong L.F., 2009. Microsatellites within genes and ESTs of the Pacific oyster Crassostrea gigas and their transferability in five other Crassostrea species. Electron. J. Biotechn. 12, DOI: 10.2225/vol12-issue3-fulltext-9.

Li, Q., Yu, H., Yu, R.H., 2006. Genetic variability assessed by microsatellites in cultured populations of the Pacific oyster (Crassostrea gigas) in China. Aquaculture 259, 95-102.

Li, R.H., Li, Q., Yu, R.H., 2009. Parentage determination and effective population size estimation in mass spawning Pacific oyster, Crassostrea gigas, based on microsatellite analysis. J. World Aquacult. Soc. 40, 667-677.

Liu, Z.J., Tan, G., Li, P., Dunham, R.A., 1999. Transcribed dinucleotide microsatellites and their associated genes from channel catfish, Ictalurus punctatus. Biochem. Biophys. Res. Commun. 259, 190-194.

Marshall, T.C., Slate, J., Kruuk, L.E.B., Pemberton, J.M., 1998. Statistical confidence for likelihood-based paternity inference in natural populations. Mol. Ecol. 7, 639-655.

Matson, S.E., Camara, M.D., Eichert, W., Banks, M.A., 2008. P-LOCl: a computer program for choosing the most efficient set of loci for parentage assignment. Mol. Ecol. Res. 8(4), 765-768.

McGoldrick, D.J., Hedgecock, D., English, L.J., Baoprasertkul, P., Ward, R.D., 2000. The transmission of microsatellite alleles in Australian and North American stocks of the Pacific oyster (Crassostrea gigas): selection and null alleles. J. Shellfish Res. 19, 779-788. 
Mori, K., 1979. Effects of artificial eutrophication on the metabolism of the Japanese oyster Crassostrea gigas. Mar. Biol. 53, 361-369.

Norris, A.T., Bradley, D.G., Cunningham, E.P., 2000. Parentage and relatedness determination in farmed Atlantic salmon (Salmo salar) using microsatellite markers. Aquaculture 182, 73-83.

Pashley, C.H., Ellis, J.R., McCauley, D.E., Burke, J.M., 2006. EST Databases as a source for molecular markers: Lessons from Helianthus. J. Hered. 97(4), 381-388.

Perdue, J., Erickson, G., 1984. A comparison of the gametogenetic cycle between the Pacific oyster Crassostrea gigas and the suminoe oyster Crassostrea rivularis in Washington state, Aquaculture 37, 231-237.

Porta, J., Porta, J.M., Martinez-Rotriguez, G., Alvarez, M.D.C., 2006. Development of a microsatellite multiplex PCR for Senegalese sole (Solea senegalensis) and its application to broodstock management. Aquaculture 56, 159-166.

Samain, J.F., McCombie, H., 2008. Summer mortality of Pacific Oyster Crassostrea gigas. Versailles, France.

Sauvage, C., Boudry, P., De Koning, D.J., Haley, C.S., Heurtebise, S., Lapègue, S., 2010. Quantitative Trait Loci for resistance to summer mortality and OsHV1 load in the Pacific oyster (Crassostrea gigas). Anim. Genet. 41, 390-399.

Sauvage, C., Boudry, P., Lapègue, S., 2009. Identification and characterization of 18 novel polymorphic microsatellite makers derived from expressed sequence tags in the Pacific oyster Crassostrea gigas. Mol. Ecol. Res. 9, 853-855.

Sellos, D., Moal, J., Degremont, L., Huvet, A., Daniel, J.Y., Nicoulaud, S., Boudry, P., Samain, J.F., Wormhoudt A.V., 2003. Structure of amylase genes in populations of Pacific cupped oyster (Crassostrea gigas): tissue expression and allelic polymorphism. Mar. Biotechnol. 5, 360-72.

Tanguy, A., Bierne, N., Saavedra, C., Pina, B., Bachère, E., Kube, M., Bazin, E., Bonhomme, F., Boudry, P., Boulo, V., Boutet, I., Cancela, L., Dossat, C., Favrel, P., Huvet, A., Jarque, S., Jollivet, D., Klages, S., Lapègue, S., Leite, R., Moal, J., Moraga, D., Reinhardt, R., Samain, J.F., Zouros, E., Canario, A., 2008. Increasing genomic information in bivalves through new EST collections in four species: Development of new genetic markers for environmental studies and genome evolution. Gene 408, 27-36.

Taris, N., Baron, S., Sharbel, T.F., Sauvage, C., Boudry, P., 2005. A combined microsatellite multiplexing and boiling DNA extraction method for high throughput parentage analyses in the Pacific Oyster (Crassostrea gigas). Aquacult. Res. 36, 516-518.

$\mathrm{Yu}, \mathrm{H} ., \mathrm{Li}, \mathrm{Q} ., 2$ 2007. EST-SSR markers from the Pacific oyster, Crassostrea gigas. Mol. Ecol. Notes 7, 860-862.

Yu, H., Li, Q., 2008. Exploiting EST Databases for the Development and Characterization of EST-SSRs in the Pacific Oyster (Crassostrea gigas). J. Hered. 99(2), 208-214. 
Table 2. Segregation analysis of microsatellite alleles in Crassostrea gigas from twelve full-families.

\begin{tabular}{|c|c|c|c|c|c|c|c|c|}
\hline Family & Multiplex & Locus & Dam & Sire & Genotypes of progeny & Expected ratio & Observes ratio & $P$ value \\
\hline \multirow[t]{12}{*}{ LOT 1} & \multirow[t]{3}{*}{ Multiplex set 1} & CGE007 & $\mathrm{A} / \mathrm{A}$ & $\mathrm{A} / \mathrm{A}$ & $\mathrm{A} / \mathrm{A}$ & 1 & 23 & - \\
\hline & & Cgsili43 & $\mathrm{A} / \mathrm{B}$ & $\mathrm{C} / \mathrm{D}$ & A/C:A/D:B/C:B/D & 1:1:1:1 & 4:9:3:7 & 0.266 \\
\hline & & Cgsili46 & $\mathrm{A} / \mathrm{A}$ & $\mathrm{B} / \mathrm{A}$ & $\mathrm{A} / \mathrm{B}: \mathrm{A} / \mathrm{A}$ & $1: 1$ & $15: 8$ & 0.144 \\
\hline & \multirow[t]{3}{*}{ Multiplex set 2} & CGE009 & $\mathrm{A} / \mathrm{B}$ & $\mathrm{A} / \mathrm{C}$ & A/A:A/C:A/B:B/C & 1:1:1:1 & $7: 8: 2: 5$ & 0.282 \\
\hline & & AMY & $\mathrm{A} / \mathrm{B}$ & $\mathrm{C} / \mathrm{A}$ & $\mathrm{A} / \mathrm{C}: \mathrm{A} / \mathrm{A}: \mathrm{B} / \mathrm{C}: \mathrm{B} / \mathrm{A}$ & 1:1:1:1 & 9:5:0:6 & 0.038 \\
\hline & & Cgsili44 & $\mathrm{A} / \mathrm{B}$ & $\mathrm{C} / \mathrm{C}$ & $\mathrm{A} / \mathrm{C}: \mathrm{B} / \mathrm{C}$ & $1: 1$ & $7: 14$ & 0.127 \\
\hline & \multirow{3}{*}{ Multiplex set 3} & Cgsili39 & $\mathrm{A} / \mathrm{X}$ & $\mathrm{B} / \mathrm{X}$ & A/B:A/X:X/B:XX & 1:1:1:1 & $20: 2: 1: 0$ & 0.000 \\
\hline & & Cgsili50 & $\mathrm{A} / \mathrm{B}$ & $\mathrm{B} / \mathrm{C}$ & A/B:A/C:B/B:B/C & 1:1:1:1 & 8:6:1:8 & 0.127 \\
\hline & & Cgsili4 & $\mathrm{A} / \mathrm{B}$ & $\mathrm{C} / \mathrm{D}$ & A/C:A/D:B/C:B/D & 1:1:1:1 & 2:3:11:7 & 0.032 \\
\hline & \multirow[t]{3}{*}{ Multiplex set 4} & CGG008 & $\mathrm{A} / \mathrm{X}$ & $\mathrm{B} / \mathrm{C}$ & A/B:A/C:X/B:X/C & 1:1:1:1 & $4: 4: 6: 4$ & 0.881 \\
\hline & & Cgsili37 & $\mathrm{A} / \mathrm{B}$ & $\mathrm{C} / \mathrm{B}$ & $\mathrm{A} / \mathrm{C}: \mathrm{A} / \mathrm{B}: \mathrm{B} / \mathrm{C}: \mathrm{B} / \mathrm{B}$ & $1: 1: 1: 1$ & 9:5:7:2 & 0.199 \\
\hline & & Cgsili6 & $\mathrm{A} / \mathrm{B}$ & $\mathrm{B} / \mathrm{X}$ & $\mathrm{A} / \mathrm{B}: \mathrm{A} / \mathrm{X}:(\mathrm{B} / \mathrm{B}+\mathrm{B} / \mathrm{X})$ & $1: 1: 2$ & $9: 1: 13$ & 0.114 \\
\hline \multirow[t]{12}{*}{ LOT 2} & \multirow[t]{3}{*}{ Multiplex set 1} & CGE007 & $\mathrm{B} / \mathrm{A}$ & $\mathrm{C} / \mathrm{A}$ & $\mathrm{B} / \mathrm{C}: \mathrm{B} / \mathrm{A}: \mathrm{A} / \mathrm{C}: \mathrm{A} / \mathrm{A}$ & 1:1:1:1 & $2: 11: 0: 9$ & 0.001 \\
\hline & & Cgsili43 & $\mathrm{A} / \mathrm{E}$ & $\mathrm{A} / \mathrm{F}$ & A/A:A/F:E/A:E/F & 1:1:1:1 & 6:8:3:6 & 0.529 \\
\hline & & Cgsili46 & $\mathrm{C} / \mathrm{A}$ & $\mathrm{C} / \mathrm{D}$ & C/C:C/D:C/A:D/A & 1:1:1:1 & $9: 3: 5: 4$ & 0.267 \\
\hline & \multirow[t]{3}{*}{ Multiplex set 2} & CGE009 & $\mathrm{D} / \mathrm{A}$ & $\mathrm{E} / \mathrm{D}$ & D/E:D/D:A/E:A/D & 1:1:1:1 & $6: 7: 6: 4$ & 0.843 \\
\hline & & AMY & $\mathrm{D} / \mathrm{E}$ & $\mathrm{F} / \mathrm{G}$ & D/F:D/G:E/F:E/G & 1:1:1:1 & $1: 1: 10: 8$ & 0.004 \\
\hline & & Cgsili44 & $\mathrm{B} / \mathrm{C}$ & $\mathrm{B} / \mathrm{B}$ & $\mathrm{B} / \mathrm{B}: \mathrm{B} / \mathrm{C}$ & $1: 1$ & $14: 8$ & 0.201 \\
\hline & Multiplex set 3 & Cgsili39 & $\mathrm{B} / \mathrm{X}$ & $\mathrm{A} / \mathrm{X}$ & B/A:B/X:X/A:X/X & 1:1:1:1 & 8:1:11:3 & 0.012 \\
\hline & & Cgsili50 & $\mathrm{A} / \mathrm{D}$ & $\mathrm{E} / \mathrm{F}$ & $\mathrm{A} / \mathrm{E}: \mathrm{A} / \mathrm{F}: \mathrm{D} / \mathrm{E}: \mathrm{D} / \mathrm{F}$ & 1:1:1:1 & 1:7:3:12 & 0.006 \\
\hline & & Cgsili4 & $\mathrm{E} / \mathrm{B}$ & $\mathrm{B} / \mathrm{F}$ & $\mathrm{E} / \mathrm{B}: \mathrm{E} / \mathrm{F}: \mathrm{B} / \mathrm{B}: \mathrm{B} / \mathrm{F}$ & 1:1:1:1 & $2: 7: 7: 7$ & 0.353 \\
\hline & Multiplex set 4 & CGG008 & $\mathrm{D} / \mathrm{B}$ & $\mathrm{E} / \mathrm{X}$ & D/E:D/X:B/E:B/X & 1:1:1:1 & $5: 9: 2: 7$ & 0.199 \\
\hline & & Cgsili37 & $\mathrm{D} / \mathrm{E}$ & F/G & D/F:D/G:E/F:E/G & 1:1:1:1 & 9:7:3:4 & 0.266 \\
\hline & & Cgsili6 & $\mathrm{C} / \mathrm{D}$ & $\mathrm{E} / \mathrm{C}$ & $\mathrm{C} / \mathrm{E}: \mathrm{C} / \mathrm{C}: \mathrm{D} / \mathrm{E}: \mathrm{D} / \mathrm{C}$ & 1:1:1:1 & 7:3:7:5 & 0.572 \\
\hline LOT 3 & Multiplex set 1 & CGE007 & $\mathrm{B} / \mathrm{D}$ & $\mathrm{E} / \mathrm{A}$ & $\mathrm{B} / \mathrm{E}: \mathrm{B} / \mathrm{A}: \mathrm{D} / \mathrm{E}: \mathrm{D} / \mathrm{A}$ & 1:1:1:1 & $7: 6: 7: 2$ & 0.378 \\
\hline & & Cgsili43 & $\mathrm{G} / \mathrm{H}$ & $\mathrm{A} / \mathrm{I}$ & G/A:G/I:H/A:H/I & 1:1:1:1 & $4: 7: 6: 4$ & 0.733 \\
\hline & & Cgsili46 & $\mathrm{D} / \mathrm{B}$ & $\mathrm{E} / \mathrm{F}$ & $\mathrm{D} / \mathrm{E}: \mathrm{D} / \mathrm{F}: \mathrm{B} / \mathrm{E}: \mathrm{B} / \mathrm{F}$ & 1:1:1:1 & $4: 4: 7: 7$ & 0.651 \\
\hline & Multiplex set 2 & CGE009 & $\mathrm{F} / \mathrm{C}$ & $\mathrm{E} / \mathrm{F}$ & F/E:F/F:C/E:C/F & 1:1:1:1 & $6: 8: 2: 6$ & 0.327 \\
\hline & & AMY & $\mathrm{E} / \mathrm{H}$ & $\mathrm{A} / \mathrm{I}$ & E/A:E/I:H/A:H/I & 1:1:1:1 & 4:3:4:10 & 0.119 \\
\hline & & Cgsili44 & $\mathrm{B} / \mathrm{D}$ & $\mathrm{D} / \mathrm{D}$ & B/D:D/D & $1: 1$ & $10: 11$ & 0.827 \\
\hline & Multiplex set 3 & Cgsili39 & $\mathrm{A} / \mathrm{C}$ & $\mathrm{D} / \mathrm{X}$ & A/D:A/X:C/D:C/X & 1:1:1:1 & 1:7:5:7 & 0.187 \\
\hline & & Cgsili50 & $\mathrm{A} / \mathrm{D}$ & $\mathrm{B} / \mathrm{B}$ & $\mathrm{A} / \mathrm{B}: \mathrm{D} / \mathrm{B}$ & $1: 1$ & $10: 8$ & 0.637 \\
\hline & & Cgsili4 & $\mathrm{G} / \mathrm{H}$ & $\mathrm{B} / \mathrm{B}$ & $\mathrm{G} / \mathrm{B}: \mathrm{H} / \mathrm{B}$ & $1: 1$ & $11: 11$ & 1.000 \\
\hline & Multiplex set 4 & CGG008 & $\mathrm{A} / \mathrm{F}$ & $\mathrm{D} / \mathrm{B}$ & A/D:A/B:F/D:F/B & 1:1:1:1 & $5: 6: 8: 3$ & 0.500 \\
\hline & & Cgsili37 & $\mathrm{H} / \mathrm{B}$ & $\mathrm{I} / \mathrm{J}$ & H/I:H/J:B/I:B/J & 1:1:1:1 & 3:9:6:4 & 0.282 \\
\hline & & Cgsili6 & $\mathrm{F} / \mathrm{X}$ & $\mathrm{F} / \mathrm{X}$ & $(F / F+F / X): X / X$ & $3: 1$ & $19: 4$ & 0.399 \\
\hline LOT 4 & Multiplex set 1 & CGE007 & $\mathrm{A} / \mathrm{A}$ & $\mathrm{E} / \mathrm{F}$ & $\mathrm{A} / \mathrm{E}: \mathrm{A} / \mathrm{F}$ & $1: 1$ & $14: 9$ & 0.297 \\
\hline & & Cgsili43 & $\mathrm{A} / \mathrm{J}$ & $\mathrm{H} / \mathrm{J}$ & A/H:A/J:J/H:J/J & 1:1:1:1 & $6: 4: 5: 6$ & 0.914 \\
\hline & & Cgsili46 & $\mathrm{C} / \mathrm{A}$ & $\mathrm{C} / \mathrm{A}$ & C/C:C/A:A/A & $1: 2: 1$ & $6: 8: 8$ & 0.572 \\
\hline & Multiplex set 2 & CGE009 & $\mathrm{E} / \mathrm{C}$ & $\mathrm{F} / \mathrm{C}$ & $\mathrm{E} / \mathrm{F}: \mathrm{E} / \mathrm{C}: \mathrm{F} / \mathrm{C}: \mathrm{C} / \mathrm{C}$ & 1:1:1:1 & $5: 7: 8: 3$ & 0.464 \\
\hline & & AMY & $\mathrm{J} / \mathrm{K}$ & $\mathrm{H} / \mathrm{K}$ & $\mathrm{J} / \mathrm{H}: \mathrm{J} / \mathrm{K}: \mathrm{K} / \mathrm{H}: \mathrm{K} / \mathrm{K}$ & 1:1:1:1 & 6:3:8:2 & 0.188 \\
\hline & & Cgsili44 & $\mathrm{C} / \mathrm{C}$ & $\mathrm{A} / \mathrm{C}$ & $\mathrm{C} / \mathrm{A}: \mathrm{C} / \mathrm{C}$ & $1: 1$ & $16: 6$ & 0.033 \\
\hline & Multiplex set 3 & Cgsili39 & $\mathrm{D} / \mathrm{X}$ & $\mathrm{E} / \mathrm{X}$ & D/E:D/X:X/E:X/X & 1:1:1:1 & 4:6:8:2 & 0.261 \\
\hline & & Cgsili50 & $\mathrm{B} / \mathrm{C}$ & $\mathrm{B} / \mathrm{D}$ & B/B:B/D:C/B:C/D & 1:1:1:1 & 4:5:8:5 & 0.651 \\
\hline & & Cgsili4 & $\mathrm{I} / \mathrm{B}$ & G/I & I/G:I/I:B/G:B/I & 1:1:1:1 & $6: 7: 5: 4$ & 0.823 \\
\hline & Multiplex set 4 & CGG008 & $\mathrm{G} / \mathrm{X}$ & $\mathrm{B} / \mathrm{G}$ & $\mathrm{G} / \mathrm{B}:(\mathrm{G} / \mathrm{G}+\mathrm{G} / \mathrm{X}): \mathrm{X} / \mathrm{B}$ & $1: 2: 1$ & $2: 16: 2$ & 0.066 \\
\hline & & Cgsili37 & $\mathrm{A} / \mathrm{B}$ & $\mathrm{A} / \mathrm{E}$ & $\mathrm{A} / \mathrm{A}: \mathrm{A} / \mathrm{E}: \mathrm{B} / \mathrm{A}: \mathrm{B} / \mathrm{E}$ & 1:1:1:1 & 8:3:7:4 & 0.378 \\
\hline & & Cgsili6 & $\mathrm{B} / \mathrm{X}$ & $\mathrm{G} / \mathrm{H}$ & $\mathrm{B} / \mathrm{G}: \mathrm{B} / \mathrm{H}: \mathrm{X} / \mathrm{G}: \mathrm{X} / \mathrm{H}$ & 1:1:1:1 & 4:2:9:4 & 0.131 \\
\hline LOT 5 & Multiplex set 1 & CGE007 & $\mathrm{G} / \mathrm{B}$ & G/A & G/G:G/A:B/G:B/A & 1:1:1:1 & $6: 6: 3: 8$ & 0.529 \\
\hline & & Cgsili43 & G/A & $\mathrm{C} / \mathrm{D}$ & G/C:G/D:A/C:A/D & 1:1:1:1 & $4: 5: 10: 3$ & 0.153 \\
\hline & & Cgsili46 & $\mathrm{A} / \mathrm{G}$ & $\mathrm{A} / \mathrm{H}$ & A/A:A/H:G/A:G/H & 1:1:1:1 & 5:5:5:8 & 0.759 \\
\hline & Multiplex set 2 & CGE009 & $\mathrm{D} / \mathrm{B}$ & $\mathrm{E} / \mathrm{D}$ & D/E:D/D:B/E:B/D & 1:1:1:1 & $6: 6: 6: 5$ & 0.988 \\
\hline & & AMY & $\mathrm{L} / \mathrm{A}$ & G/I & L/G:L/I:A/G:A/I & 1:1:1:1 & $5: 4: 6: 7$ & 0.823 \\
\hline & & Cgsili44 & $\mathrm{A} / \mathrm{D}$ & $\mathrm{C} / \mathrm{C}$ & $\mathrm{A} / \mathrm{C}: \mathrm{D} / \mathrm{C}$ & $1: 1$ & $7: 16$ & 0.061 \\
\hline & Multiplex set 3 & Cgsili39 & $\mathrm{F} / \mathrm{G}$ & $\mathrm{A} / \mathrm{X}$ & F/A:F/X:G/A:G/X & $1: 1: 1: 1$ & 8:6:3:5 & 0.500 \\
\hline & & Cgsili50 & $\mathrm{B} / \mathrm{D}$ & $\mathrm{B} / \mathrm{C}$ & B/B:B/C:D/B:D/C & 1:1:1:1 & 3:3:9:8 & 0.148 \\
\hline & & Cgsili4 & $\mathrm{B} / \mathrm{J}$ & $\mathrm{C} / \mathrm{K}$ & B/C:B/K:J/C:J/K & 1:1:1:1 & $5: 8: 6: 4$ & 0.677 \\
\hline & Multiplex set 4 & CGG008 & $\mathrm{H} / \mathrm{X}$ & $\mathrm{B} / \mathrm{C}$ & H/B:H/C:X/B:X/C & 1:1:1:1 & $5: 4: 4: 10$ & 0.23 \\
\hline & & Cgsili37 & $\mathrm{C} / \mathrm{B}$ & $\mathrm{B} / \mathrm{J}$ & $\mathrm{C} / \mathrm{B}: \mathrm{C} / \mathrm{J}: \mathrm{B} / \mathrm{B}: \mathrm{B} / \mathrm{J}$ & 1:1:1:1 & $4: 7: 5: 7$ & 0.759 \\
\hline & & Cgsili6 & $\mathrm{E} / \mathrm{X}$ & $\mathrm{X} / \mathrm{X}$ & $\mathrm{E} / \mathrm{X}: \mathrm{X} / \mathrm{X}$ & $1: 1$ & $10: 13$ & 0.532 \\
\hline LOT 6 & Multiplex set 1 & CGE007 & $\mathrm{A} / \mathrm{A}$ & F/A & $\mathrm{A} / \mathrm{F}: \mathrm{A} / \mathrm{A}$ & $1: 1$ & 14:9 & 0.297 \\
\hline & & Cgsili43 & $\mathrm{H} / \mathrm{B}$ & $\mathrm{A} / \mathrm{J}$ & H/A:H/J:B/A:B/J & 1:1:1:1 & $8: 7: 1: 7$ & 0.148 \\
\hline & & Cgsili46 & $\mathrm{C} / \mathrm{B}$ & $\mathrm{A} / \mathrm{A}$ & $\mathrm{C} / \mathrm{A}: \mathrm{B} / \mathrm{A}$ & $1: 1$ & $9: 14$ & 0.297 \\
\hline & Multiplex set 2 & CGE009 & $\mathrm{D} / \mathrm{A}$ & $\mathrm{D} / \mathrm{C}$ & D/D:D/C:A/D:A/C & 1:1:1:1 & 4:7:6:6 & 0.843 \\
\hline & & AMY & $\mathrm{C} / \mathrm{M}$ & $\mathrm{E} / \mathrm{K}$ & C/E:C/K:M/E:M/K & 1:1:1:1 & $7: 4: 2: 9$ & 0.153 \\
\hline & & Cgsili44 & $\mathrm{B} / \mathrm{D}$ & $\mathrm{A} / \mathrm{B}$ & B/A:B/B:D/A:D/B & 1:1:1:1 & 5:6:2:9 & 0.208 \\
\hline & Multiplex set 3 & Cgsili39 & $\mathrm{B} / \mathrm{D}$ & $\mathrm{E} / \mathrm{F}$ & B/E:B/F:D/E:D/F & 1:1:1:1 & $6: 3: 10: 4$ & 0.172 \\
\hline & & Cgsili50 & $\mathrm{B} / \mathrm{G}$ & $\mathrm{A} / \mathrm{E}$ & B/A:B/E:G/A:G/E & 1:1:1:1 & $8: 1: 11: 3$ & 0.012 \\
\hline & & Cgsili4 & G/B & $\mathrm{E} / \mathrm{B}$ & G/E:G/B:B/E:B/B & 1:1:1:1 & $8: 7: 5: 2$ & 0.282 \\
\hline & Multiplex set 4 & CGG008 & $\mathrm{I} / \mathrm{B}$ & $\mathrm{A} / \mathrm{G}$ & I/A:I/G:B/A:B/G & 1:1:1:1 & $6: 7: 5: 5$ & 0.924 \\
\hline & & Cgsili37 & $\mathrm{K} / \mathrm{E}$ & $\mathrm{L} / \mathrm{B}$ & K/L:K/B:E/L:E/B & 1:1:1:1 & 9:4:6:4 & 0.405 \\
\hline & & Cgsili6 & $\mathrm{G} / \mathrm{I}$ & $\mathrm{B} / \mathrm{J}$ & G/B:G/J:I/B:I/J & 1:1:1:1 & 8:10:3:2 & 0.051 \\
\hline
\end{tabular}


Table 2 (continued)

\begin{tabular}{|c|c|c|c|c|c|c|c|c|}
\hline Family & Multiplex & Locus & Dam & Sire & Genotypes of progeny & Expected ratio & Observes ratio & $P$ value \\
\hline \multirow[t]{12}{*}{ LOT 7} & \multirow[t]{3}{*}{ Multiplex set 1} & CGE007 & $\mathrm{H} / \mathrm{A}$ & $\mathrm{A} / \mathrm{A}$ & H/A:A/A & $1: 1$ & $16: 7$ & 0.061 \\
\hline & & Cgsili43 & $\mathrm{A} / \mathrm{J}$ & $\mathrm{G} / \mathrm{K}$ & A/G:A/K:J/G:J/K & $1: 1: 1: 1$ & 3:8:3:9 & 0.148 \\
\hline & & Cgsili46 & $\mathrm{A} / \mathrm{H}$ & $\mathrm{A} / \mathrm{G}$ & A/A:A/G:H/A:H/G & 1:1:1:1 & 2:9:3:9 & 0.059 \\
\hline & \multirow[t]{3}{*}{ Multiplex set 2} & CGE009 & $\mathrm{A} / \mathrm{C}$ & $\mathrm{A} / \mathrm{B}$ & A/A:A/B:C/A:C/B & 1:1:1:1 & $4: 5: 7: 7$ & 0.759 \\
\hline & & AMY & $\mathrm{N} / \mathrm{O}$ & $\mathrm{A} / \mathrm{K}$ & N/A:N/K:O/A:O/K & 1:1:1:1 & 3:5:5:9 & 0.327 \\
\hline & & Cgsili44 & $\mathrm{B} / \mathrm{D}$ & $\mathrm{D} / \mathrm{C}$ & B/D:B/C:D/D:D/C & 1:1:1:1 & $4: 6: 5: 8$ & 0.677 \\
\hline & \multirow[t]{3}{*}{ Multiplex set 3} & Cgsili39 & $\mathrm{A} / \mathrm{X}$ & $\mathrm{A} / \mathrm{G}$ & $(\mathrm{A} / \mathrm{A}+\mathrm{X} / \mathrm{A}): \mathrm{A} / \mathrm{G}: \mathrm{X} / \mathrm{G}$ & $2: 1: 1$ & 10:4:9 & 0.464 \\
\hline & & Cgsili50 & $\mathrm{A} / \mathrm{B}$ & $\mathrm{A} / \mathrm{B}$ & $\mathrm{A} / \mathrm{A}: \mathrm{A} / \mathrm{B}: \mathrm{B} / \mathrm{B}$ & $1: 2: 1$ & $4: 10: 9$ & 0.464 \\
\hline & & Cgsili4 & $\mathrm{I} / \mathrm{B}$ & $\mathrm{J} / \mathrm{L}$ & $\mathrm{I} / \mathrm{J}: \mathrm{I} / \mathrm{L}: \mathrm{B} / \mathrm{J}: \mathrm{B} / \mathrm{L}$ & 1:1:1:1 & $6: 6: 5: 6$ & 0.989 \\
\hline & \multirow[t]{3}{*}{ Multiplex set 4} & CGG008 & $\mathrm{X} / \mathrm{X}$ & $\mathrm{E} / \mathrm{A}$ & $\mathrm{X} / \mathrm{E}: \mathrm{X} / \mathrm{A}$ & $1: 1$ & $12: 10$ & 0.670 \\
\hline & & Cgsili37 & $\mathrm{I} / \mathrm{B}$ & $\mathrm{B} / \mathrm{M}$ & I/B:I/M:B/B:B/M & 1:1:1:1 & 7:9:7:0 & 0.043 \\
\hline & & Cgsili6 & $\mathrm{E} / \mathrm{B}$ & C/D & $\mathrm{E} / \mathrm{C}: \mathrm{E} / \mathrm{D}: \mathrm{B} / \mathrm{C}: \mathrm{B} / \mathrm{D}$ & 1:1:1:1 & 8:8:4:3 & 0.307 \\
\hline \multirow[t]{12}{*}{ LOT 8} & \multirow[t]{3}{*}{ Multiplex set 1} & CGE007 & $\mathrm{E} / \mathrm{A}$ & $\mathrm{F} / \mathrm{B}$ & E/F:E/B:A/F:A/B & 1:1:1:1 & 8:4:4:6 & 0.572 \\
\hline & & Cgsili43 & $\mathrm{A} / \mathrm{L}$ & $\mathrm{C} / \mathrm{C}$ & $\mathrm{A} / \mathrm{C}: \mathrm{L} / \mathrm{C}$ & $1: 1$ & 9:13 & 0.394 \\
\hline & & Cgsili46 & $\mathrm{A} / \mathrm{A}$ & $\mathrm{B} / \mathrm{A}$ & $\mathrm{A} / \mathrm{B}: \mathrm{A} / \mathrm{A}$ & $1: 1$ & $12: 10$ & 0.670 \\
\hline & \multirow[t]{3}{*}{ Multiplex set 2} & CGE009 & $\mathrm{A} / \mathrm{C}$ & $\mathrm{D} / \mathrm{B}$ & A/D:A/B:C/D:C/B & 1:1:1:1 & 9:2:7:5 & 0.199 \\
\hline & & AMY & $\mathrm{E} / \mathrm{G}$ & $\mathrm{A} / \mathrm{O}$ & E/A:E/O:G/A:G/O & 1:1:1:1 & 3:9:4:7 & 0.266 \\
\hline & & Cgsili44 & $\mathrm{C} / \mathrm{C}$ & $\mathrm{A} / \mathrm{D}$ & $\mathrm{C} / \mathrm{A}: \mathrm{C} / \mathrm{D}$ & $1: 1$ & $8: 14$ & 0.201 \\
\hline & Multiplex set 3 & Cgsili39 & $\mathrm{A} / \mathrm{C}$ & $\mathrm{C} / \mathrm{X}$ & A/C:A/X:(C/C + C/X) & $1: 1: 2$ & $0: 7: 15$ & 0.061 \\
\hline & & Cgsili50 & $\mathrm{C} / \mathrm{D}$ & $\mathrm{B} / \mathrm{B}$ & $\mathrm{C} / \mathrm{B}: \mathrm{D} / \mathrm{B}$ & $1: 1$ & $7: 15$ & 0.088 \\
\hline & & Cgsili4 & $\mathrm{B} / \mathrm{J}$ & $\mathrm{G} / \mathrm{G}$ & $\mathrm{B} / \mathrm{G}: \mathrm{J} / \mathrm{G}$ & $1: 1$ & $3: 11$ & 0.033 \\
\hline & Multiplex set 4 & CGG008 & $\mathrm{E} / \mathrm{X}$ & $\mathrm{H} / \mathrm{F}$ & $\mathrm{E} / \mathrm{H}: \mathrm{E} / \mathrm{F}: \mathrm{X} / \mathrm{H}: \mathrm{X} / \mathrm{F}$ & 1:1:1:1 & $9: 4: 6: 4$ & 0.405 \\
\hline & & Cgsili37 & N/O & $\mathrm{H} / \mathrm{L}$ & N/H:N/L:O/H:O/L & 1:1:1:1 & $1: 5: 4: 13$ & 0.003 \\
\hline & & Cgsili6 & $\mathrm{X} / \mathrm{X}$ & $\mathrm{B} / \mathrm{X}$ & $\mathrm{X} / \mathrm{B}: \mathrm{X} / \mathrm{X}$ & $1: 1$ & $5: 18$ & 0.007 \\
\hline LOT 9 & Multiplex set 1 & CGE007 & $\mathrm{B} / \mathrm{A}$ & G/B & B/G:B/B:A/G:A/B & 1:1:1:1 & $7: 2: 10: 4$ & 0.094 \\
\hline & & Cgsili43 & $\mathrm{A} / \mathrm{C}$ & $\mathrm{G} / \mathrm{A}$ & $\mathrm{A} / \mathrm{G}: \mathrm{A} / \mathrm{A}: \mathrm{C} / \mathrm{G}: \mathrm{C} / \mathrm{A}$ & $1: 1: 1: 1$ & $3: 2: 5: 10$ & 0.055 \\
\hline & & Cgsili46 & $\mathrm{B} / \mathrm{A}$ & $\mathrm{B} / \mathrm{E}$ & B/B:B/E:A/B:A/E & 1:1:1:1 & $5: 10: 4: 3$ & 0.153 \\
\hline & Multiplex set 2 & CGE009 & $\mathrm{D} / \mathrm{G}$ & $\mathrm{D} / \mathrm{F}$ & D/D:D/F:G/D:G/F & $1: 1: 1: 1$ & $3: 5: 9: 5$ & 0.327 \\
\hline & & AMY & $\mathrm{P} / \mathrm{K}$ & $\mathrm{A} / \mathrm{H}$ & P/A:P/H:K/A:K/H & $1: 1: 1: 1$ & $2: 3: 7: 5$ & 0.325 \\
\hline & & Cgsili44 & $\mathrm{B} / \mathrm{D}$ & $\mathrm{A} / \mathrm{D}$ & B/A:B/D:D/A:D/D & 1:1:1:1 & $7: 5: 4: 7$ & 0.759 \\
\hline & Multiplex set 3 & Cgsili39 & $\mathrm{A} / \mathrm{X}$ & $\mathrm{A} / \mathrm{G}$ & $(\mathrm{A} / \mathrm{A}+\mathrm{X} / \mathrm{A}): \mathrm{A} / \mathrm{G}: \mathrm{X} / \mathrm{G}$ & $2: 1: 1$ & $4: 12: 7$ & 0.008 \\
\hline & & Cgsili50 & $\mathrm{B} / \mathrm{B}$ & $\mathrm{A} / \mathrm{C}$ & $\mathrm{B} / \mathrm{A}: \mathrm{B} / \mathrm{C}$ & $1: 1$ & $13: 10$ & 0.532 \\
\hline & & Cgsili4 & $\mathrm{B} / \mathrm{K}$ & $\mathrm{B} / \mathrm{J}$ & B/B:B/J:K/B:K/J & 1:1:1:1 & 2:4:9:5 & 0.158 \\
\hline & Multiplex set 4 & CGG008 & $\mathrm{H} / \mathrm{I}$ & $\mathrm{E} / \mathrm{H}$ & H/E:H/H:I/E:I/H & 1:1:1:1 & $8: 2: 6: 3$ & 0.188 \\
\hline & & Cgsili37 & $\mathrm{B} / \mathrm{N}$ & $\mathrm{C} / \mathrm{B}$ & B/C:B/B:N/C:N/B & 1:1:1:1 & $8: 4: 4: 5$ & 0.563 \\
\hline & & Cgsili6 & $\mathrm{A} / \mathrm{B}$ & $\mathrm{K} / \mathrm{C}$ & $\mathrm{A} / \mathrm{K}: \mathrm{A} / \mathrm{C}: \mathrm{B} / \mathrm{K}: \mathrm{B} / \mathrm{C}$ & 1:1:1:1 & 4:8:4:4 & 0.494 \\
\hline LOT 10 & Multiplex set 1 & CGE007 & $\mathrm{I} / \mathrm{A}$ & $\mathrm{E} / \mathrm{A}$ & I/E:I/A:A/E:A/A & 1:1:1:1 & 3:9:3:8 & 0.148 \\
\hline & & Cgsili43 & $\mathrm{M} / \mathrm{A}$ & $\mathrm{G} / \mathrm{N}$ & M/G:M/N:A/G:A/N & 1:1:1:1 & 10:8:1:3 & 0.022 \\
\hline & & Cgsili46 & $\mathrm{C} / \mathrm{A}$ & $\mathrm{B} / \mathrm{G}$ & C/B:C/G:A/B:A/G & 1:1:1:1 & $3: 5: 7: 8$ & 0.464 \\
\hline & Multiplex set 2 & CGE009 & $\mathrm{D} / \mathrm{F}$ & $\mathrm{H} / \mathrm{A}$ & D/H:D/A:F/H:F/A & 1:1:1:1 & $6: 7: 5: 5$ & 0.924 \\
\hline & & AMY & $\mathrm{H} / \mathrm{K}$ & $\mathrm{A} / \mathrm{K}$ & H/A:H/K:K/A:K/K & 1:1:1:1 & $4: 8: 7: 4$ & 0.529 \\
\hline & & Cgsili44 & $\mathrm{B} / \mathrm{B}$ & $\mathrm{A} / \mathrm{C}$ & $\mathrm{B} / \mathrm{A}: \mathrm{B} / \mathrm{C}$ & $1: 1$ & $15: 8$ & 0.144 \\
\hline & Multiplex set 3 & Cgsili39 & $\mathrm{A} / \mathrm{H}$ & $\mathrm{F} / \mathrm{G}$ & $\mathrm{A} / \mathrm{F}: \mathrm{A} / \mathrm{G}: \mathrm{H} / \mathrm{F}: \mathrm{H} / \mathrm{G}$ & 1:1:1:1 & $2: 12: 2: 7$ & 0.008 \\
\hline & & Cgsili50 & $\mathrm{C} / \mathrm{H}$ & $\mathrm{A} / \mathrm{B}$ & C/A:C/B:H/A:H/B & 1:1:1:1 & $10: 4: 4: 5$ & 0.230 \\
\hline & & Cgsili4 & $\mathrm{M} / \mathrm{N}$ & $\mathrm{J} / \mathrm{O}$ & M/J:M/O:N/J:N/O & 1:1:1:1 & $8: 8: 2: 3$ & 0.119 \\
\hline & Multiplex set 4 & CGG008 & $\mathrm{I} / \mathrm{F}$ & $\mathrm{I} / \mathrm{G}$ & I/I:I/G:F/I:F/G & 1:1:1:1 & 7:5:5:6 & 0.924 \\
\hline & & Cgsili37 & $\mathrm{F} / \mathrm{M}$ & $\mathrm{D} / \mathrm{N}$ & F/D:F/N:M/D:M/N & 1:1:1:1 & $4: 7: 4: 8$ & 0.529 \\
\hline & & Cgsili6 & $\mathrm{G} / \mathrm{X}$ & $\mathrm{B} / \mathrm{X}$ & G/B:G/X:X/B:X/X & 1:1:1:1 & $4: 15: 4: 0$ & 0.000 \\
\hline LOT11 & Multiplex set 1 & CGE007 & G/A & G/A & G/G:G/A:A/A & $1: 2: 1$ & $7: 10: 6$ & 0.924 \\
\hline & & Cgsili43 & $\mathrm{H} / \mathrm{D}$ & $\mathrm{O} / \mathrm{C}$ & H/O:H/C:D/O:D/C & 1:1:1:1 & $6: 5: 5: 7$ & 0.924 \\
\hline & & Cgsili46 & $\mathrm{C} / \mathrm{G}$ & G/E & C/G:C/E:G/G:G/E & 1:1:1:1 & 7:9:4:3 & 0.266 \\
\hline & Multiplex set 2 & CGE009 & $\mathrm{A} / \mathrm{C}$ & $\mathrm{C} / \mathrm{B}$ & A/C:A/B:C/C:C/B & 1:1:1:1 & $6: 7: 6: 4$ & 0.843 \\
\hline & & AMY & N/Q & $\mathrm{A} / \mathrm{K}$ & N/A:N/K:Q/A:Q/K & 1:1:1:1 & $6: 6: 3: 4$ & 0.701 \\
\hline & & Cgsili44 & $\mathrm{A} / \mathrm{C}$ & $\mathrm{B} / \mathrm{D}$ & A/B:A/D:C/B:C/D & 1:1:1:1 & $6: 6: 7: 4$ & 0.843 \\
\hline & Multiplex set 3 & Cgsili39 & $\mathrm{E} / \mathrm{E}$ & $\mathrm{E} / \mathrm{C}$ & $\mathrm{E} / \mathrm{E}: \mathrm{E} / \mathrm{C}$ & $1: 1$ & $23: 0$ & 0.000 \\
\hline & & Cgsili50 & $\mathrm{F} / \mathrm{H}$ & $\mathrm{A} / \mathrm{B}$ & F/A:F/B:H/A:H/B & 1:1:1:1 & 7:3:3:10 & 0.110 \\
\hline & & Cgsili4 & $\mathrm{P} / \mathrm{C}$ & $\mathrm{O} / \mathrm{O}$ & $\mathrm{P} / \mathrm{O}: \mathrm{C} / \mathrm{O}$ & $1: 1$ & $11: 12$ & 0.835 \\
\hline & Multiplex set 4 & CGG008 & $\mathrm{F} / \mathrm{C}$ & $\mathrm{I} / \mathrm{I}$ & F/I:C/I & $1: 1$ & $12: 11$ & 0.835 \\
\hline & & Cgsili37 & $\mathrm{C} / \mathrm{N}$ & $\mathrm{H} / \mathrm{N}$ & C/H:C/N:N/H:N/N & 1:1:1:1 & $3: 10: 3: 7$ & 0.110 \\
\hline & & Cgsili6 & $\mathrm{L} / \mathrm{I}$ & $\mathrm{M} / \mathrm{B}$ & L/M:L/B:I/M:I/B & 1:1:1:1 & $10: 5: 5: 3$ & 0.199 \\
\hline LOT 12 & Multiplex set 1 & CGE007 & $\mathrm{J} / \mathrm{J}$ & $\mathrm{E} / \mathrm{A}$ & J/E:J/A & $1: 1$ & $7: 5$ & 0.564 \\
\hline & & Cgsili43 & $\mathrm{B} / \mathrm{P}$ & $\mathrm{K} / \mathrm{J}$ & B/K:B/J:P/K:P/J & 1:1:1:1 & 6:3:9:5 & 0.353 \\
\hline & & Cgsili46 & I/A & $\mathrm{A} / \mathrm{G}$ & I/A:I/G:A/A:A/G & 1:1:1:1 & 8:3:4:8 & 0.307 \\
\hline & Multiplex set 2 & CGE009 & $\mathrm{E} / \mathrm{C}$ & $\mathrm{H} / \mathrm{A}$ & E/H:E/A:C/H:C/A & 1:1:1:1 & 2:8:8:5 & 0.230 \\
\hline & & AMY & $\mathrm{Q} / \mathrm{O}$ & $\mathrm{C} / \mathrm{G}$ & $\mathrm{Q} / \mathrm{C}: \mathrm{Q} / \mathrm{G}: \mathrm{O} / \mathrm{C}: \mathrm{O} / \mathrm{G}$ & 1:1:1:1 & $6: 6: 8: 2$ & 0.327 \\
\hline & & Cgsili44 & $\mathrm{C} / \mathrm{C}$ & $\mathrm{B} / \mathrm{D}$ & $\mathrm{C} / \mathrm{B}: \mathrm{C} / \mathrm{D}$ & $1: 1$ & $9: 13$ & 0.394 \\
\hline & Multiplex set 3 & Cgsili39 & $\mathrm{C} / \mathrm{E}$ & $\mathrm{D} / \mathrm{E}$ & C/D:C/E:E/D:E/E & 1:1:1:1 & 8:15:0:0 & 0.000 \\
\hline & & Cgsili50 & $\mathrm{A} / \mathrm{C}$ & $\mathrm{A} / \mathrm{D}$ & A/A:A/D:C/A:C/D & 1:1:1:1 & $7: 4: 5: 6$ & 0.823 \\
\hline & & Cgsili4 & $\mathrm{B} / \mathrm{Q}$ & $\mathrm{I} / \mathrm{L}$ & B/I:B/L:Q/I:Q/L & 1:1:1:1 & $2: 7: 5: 9$ & 0.199 \\
\hline & Multiplex set 4 & CGG008 & $\mathrm{G} / \mathrm{C}$ & $\mathrm{F} / \mathrm{X}$ & G/F:G/X:C/F:C/X & 1:1:1:1 & 3:1:11:7 & 0.013 \\
\hline & & Cgsili37 & $\mathrm{F} / \mathrm{P}$ & $\mathrm{D} / \mathrm{B}$ & F/D:F/B:P/D:P/B & 1:1:1:1 & $7: 8: 5: 3$ & 0.464 \\
\hline & & Cgsili6 & N/B & $\mathrm{C} / \mathrm{G}$ & N/C:N/G:B/C:B/G & $1: 1: 1: 1$ & 4:4:5:9 & 0.378 \\
\hline
\end{tabular}




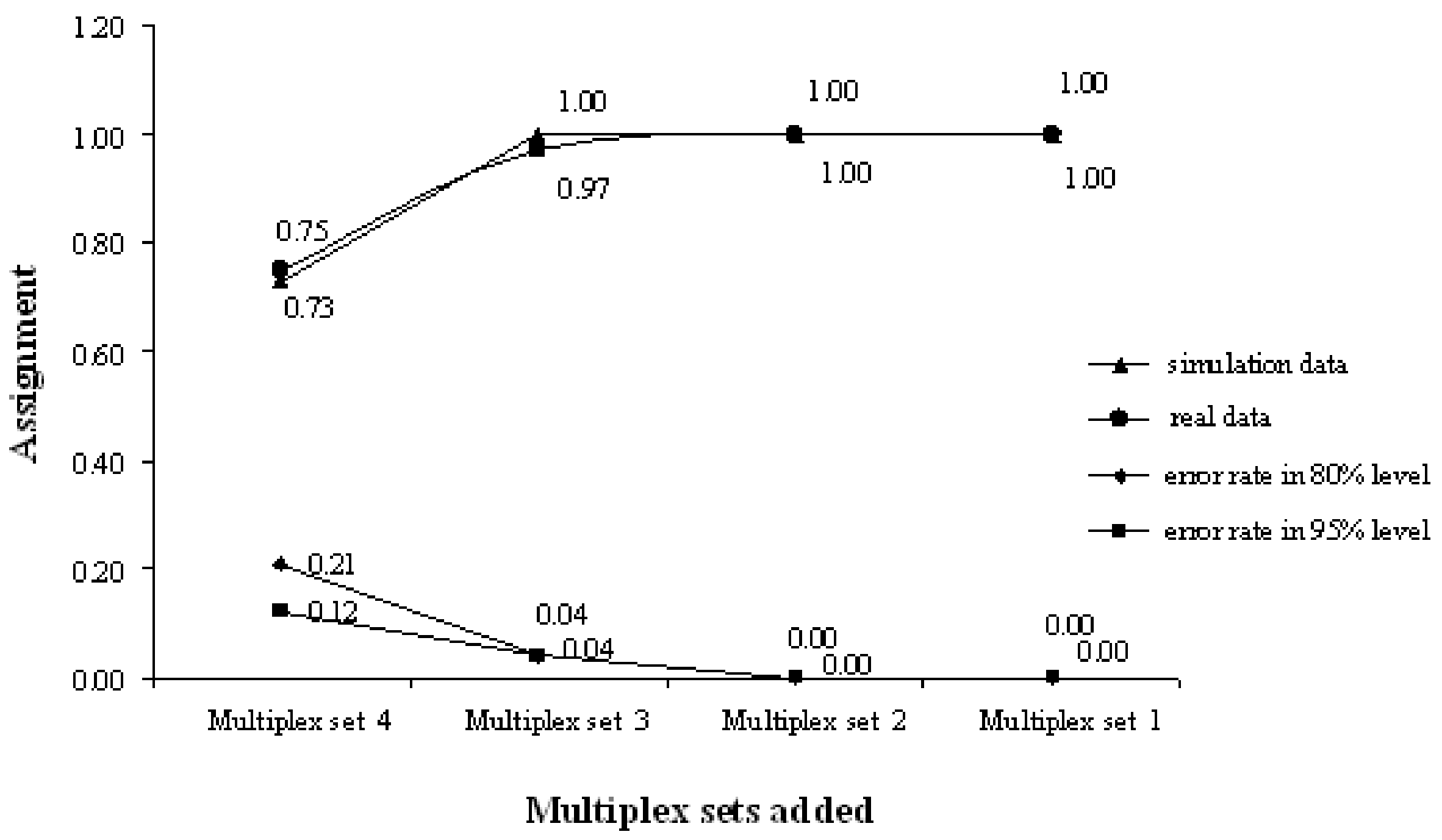


Fig. 1. Cumulative assignment success rates of simulated and real genotype data in a strict level of 95\% confidence interval. The error rates were calculated in $95 \%$ and $80 \%$ confidence interval comparing with the family data. The multiplex sets were added from the most to the least average PIC value. 


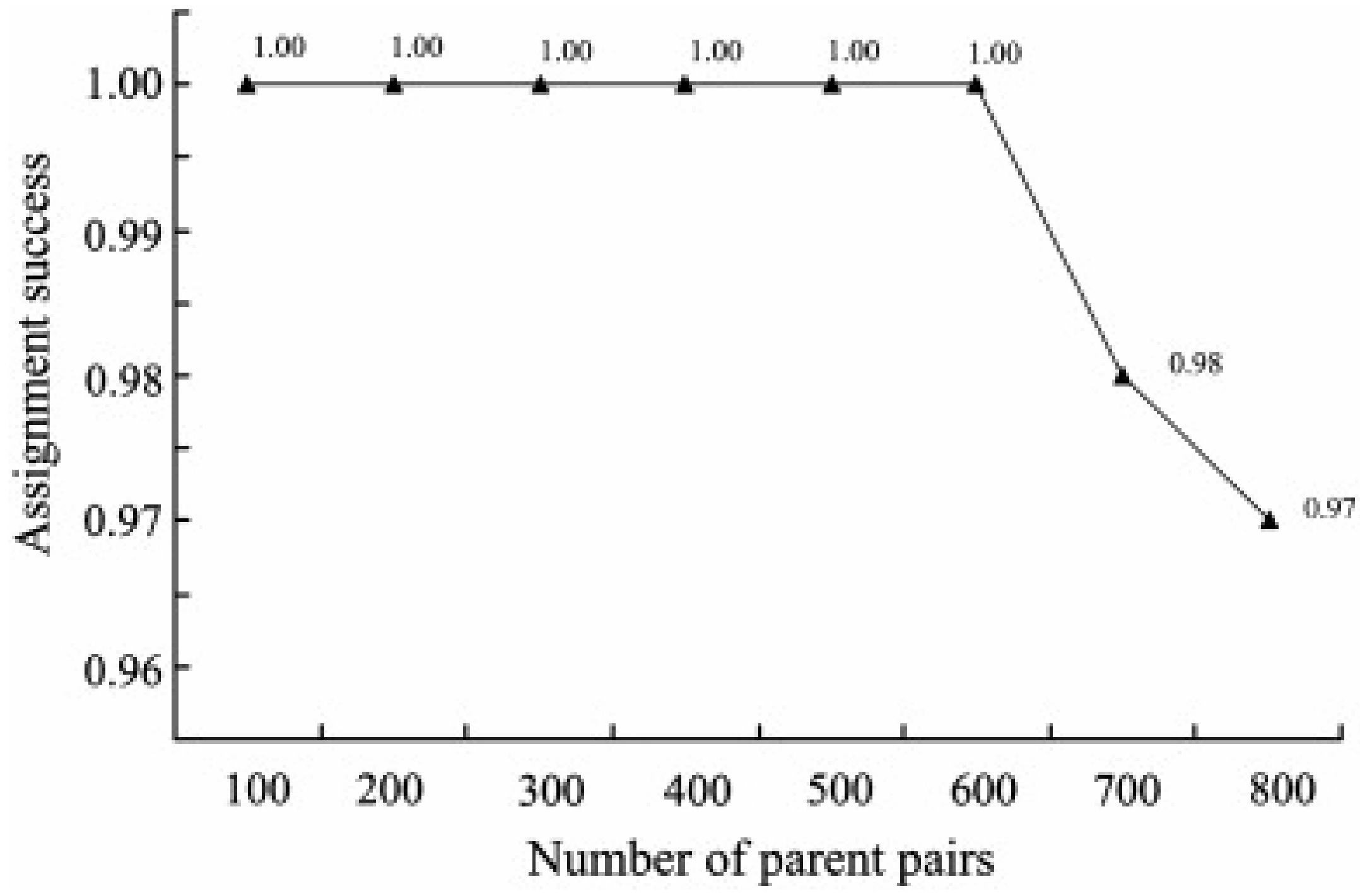

Fig. 2. Simulated assignment success rates of 10,000 offspring in parentage under a number of candidate parents from 100 to 800 with the most Polymorphic three multiplex PCRs in a strict level of $95 \%$ confidence interval. 
Table 1. Characteristics of the four EST-SSR multiplex PCRs in Crassostrea gigas .

\begin{tabular}{|c|c|c|c|c|c|c|c|c|c|c|c|c|}
\hline Locus & Repeat motif & Primer sequence $\left(5^{\prime}-3^{\prime}\right)$ & $\begin{array}{l}T_{\mathrm{a}} \\
(\mathrm{C})\end{array}$ & $\begin{array}{l}\text { Size range } \\
\text { (bp) }\end{array}$ & $\begin{array}{l}\text { Concentration } \\
\text { in multiplex- } \\
\text { PCR }(\mu \mathrm{M})\end{array}$ & $N_{\text {a }}$ & $A_{\mathrm{r}}$ & PCI & NE-1P & $\mathrm{NE}-2 \mathrm{P}^{\mathrm{b}}$ & $\mathrm{NE}-\mathrm{PP}^{\mathrm{c}}$ & $\begin{array}{l}\text { GenBank } \\
\text { Accession } \\
\text { no. }\end{array}$ \\
\hline PCR multiplex set 1 & & & 58 & & & & & & & & & \\
\hline CGE007 & $(\mathrm{TA})_{7}$ & $\begin{array}{l}\text { F: TTTCCCCTGAGAAGACCC } \\
\text { R: NED-AACCAАATCCATTCAАCATAAC }\end{array}$ & & $96-142$ & 0,1 & 10 & 9,7 & 0,702 & 0,650 & 0,462 & 0,251 & BQ427084 \\
\hline Cgsili43 & $(\mathrm{GA})_{10}$ & $\begin{array}{l}\text { F: HEX-AAATGCTGCAGAAATAATCC } \\
\text { R: AGATGGCTACAGTGAAATGG }\end{array}$ & & $210-348$ & 0,3 & 16 & 15,4 & 0,874 & 0,370 & 0,227 & 0,076 & AM854072 \\
\hline Cgsili46 & $(\mathrm{TG})_{5} \mathrm{~A}(\mathrm{GT})_{7}$ & $\begin{array}{l}\text { F: FAM-CATGACAATCGAGTCCATAA } \\
\text { R: CATGGTGGAGAAAGAGTTGT }\end{array}$ & & $165-211$ & 0,09 & 9 & 8,8 & 0,753 & 0,588 & 0,406 & 0,210 & AM856490 \\
\hline PCR multiplex set 2 & & & 52 & & & & & & & & & \\
\hline CGE009 & $(\mathrm{AG})_{7}$ & $\begin{array}{l}\text { F: TTCGTTGAAGGTGACAAGTG } \\
\text { R: NED-GCATTTTGGGATGAACAGA }\end{array}$ & & $114-128$ & 0,1 & 8 & 7,9 & 0,814 & 0,501 & 0,330 & 0,157 & CX068958 \\
\hline AMY & $(\mathrm{TC})_{37}$ & $\begin{array}{l}\text { F: HEX-ACCGGTATTGCCCGAGTTACAA } \\
\text { R: AGTTAGGCATCCCCCATTGTTC }\end{array}$ & & 199-369 & 0,2 & 17 & 16,4 & 0,892 & 0,331 & 0,198 & 0,061 & Y08370 \\
\hline Cgsili44 & $(\mathrm{AG})_{7} \mathrm{AAA}(\mathrm{GA})_{4}$ & $\begin{array}{l}\text { F: FAM-TGGCATTTCATGGTTAATTT } \\
\text { R: TGTTGTATGAAATGTCGGAA }\end{array}$ & & $349-355$ & 0,1 & 4 & 4,0 & 0,690 & 0,685 & 0,511 & 0,337 & AM858556 \\
\hline PCR multiplex set 3 & & & 58 & & & & & & & & & \\
\hline Cgsili39 & $(\mathrm{AG})_{13}$ & $\begin{array}{l}\text { F: FAM-GACCATACAGCTCTGTCCAT } \\
\text { R: GCTACTGAATGAGAATGGCT }\end{array}$ & & $355-383$ & 0,09 & 8 & 7,9 & 0,801 & 0,519 & 0,345 & 0,164 & AM854746 \\
\hline Cgsili50 & $(\mathrm{CA})_{10}$ & $\begin{array}{l}\text { F: FAM-CTATCTGAGCACGCTTCTCT } \\
\text { R: TCTCTGTCAGATGATCTCAGG }\end{array}$ & & 201-233 & 0,09 & 8 & 7,9 & 0,750 & 0,595 & 0,416 & 0,227 & AM865904 \\
\hline Cgsili4 & $(\mathrm{AG})_{26}$ & $\begin{array}{l}\text { F: HEX-GGTGCAGTAGTTGGAAACAT } \\
\text { R: TCACATTTAACTAGCGCTCTC }\end{array}$ & & 227-349 & 0,24 & 17 & 16,3 & 0,860 & 0,396 & 0,246 & 0,085 & AM854894 \\
\hline PCR multiplex set 4 & & & 58 & & & & & & & & & \\
\hline CGG008 & $(\mathrm{AG})_{20}$ & $\begin{array}{l}\text { F: TCTCСТСТАССCCGACAG } \\
\text { R: NED-GTGATGAACAAACCACCAAC }\end{array}$ & & $181-253$ & 0,4 & 9 & 9,0 & 0,870 & 0,392 & 0,242 & 0,091 & AJ579915 \\
\hline Cgsili37 & $(\mathrm{TC})_{15}$ & $\begin{array}{l}\text { F: FAM-TTGCTGGTTGTGATGAATAG } \\
\text { R: ATATCTGGCCTAACATGTGC }\end{array}$ & & $159-293$ & 0,15 & 16 & 15,6 & 0,890 & 0,335 & 0,201 & 0,061 & BQ427164 \\
\hline Cgsili6 & $(\mathrm{GA})_{26}$ & $\begin{array}{l}\text { F: HEX-ATGAACGTCCAAGTTCAGAC } \\
\text { R: ACACATTTCCTTATAAAGCC }\end{array}$ & & $270-442$ & 0,2 & 14 & 14,0 & 0,834 & 0,449 & 0,287 & 0,112 & AM854296 \\
\hline
\end{tabular}

Number of alleles $\left(N_{\mathrm{a}}\right)$, allelic richness $\left(A_{\mathrm{r}}\right)$, polymorphic information content (PIC), and average non-exclusion probability of each locus surveyed in the 24 C. gigas parents. 
The fluorescent labels were indicated by bold letters in front of the primer sequences.

a Average non-exclusion probability for one candidate parent.

b Average non-exclusion probability for one candidate parent given the genotype of a known parent of the opposite sex.

c Average non-exclusion probability for a candidate parent pair. 